\title{
Stochastic modeling of fatigue crack propagation
}

\author{
Asok Ray ${ }^{a, *}$, Sekhar Tangirala ${ }^{a}$, Shashi Phoha ${ }^{b}$ \\ ${ }^{a}$ Mechanical Engineering Department, Pennsylvania State University, University Park, PA 16802-1412, USA \\ b Information Systems Division, Applied Research Laboratory, Pennsylvania State University, University Park, \\ PA 16802-1412, USA
}

Received 29 April 1997; received in revised form 27 November 1997; accepted 18 December 1997

\begin{abstract}
This paper presents a stochastic model of fatigue-induced crack propagation in metallic materials. The crack growth rate predicted by the model is guaranteed to be non-negative. The model structure is built upon the underlying principle of Karhunen-Loève expansion and does not require solutions of stochastic differential equations in either Wiener integral or Itô integral setting. As such this crack propagation model can be readily adapted to damage monitoring and remaining life prediction of stressed structures. The model results have been verified by comparison with experimental data of time-dependent fatigue crack statistics for 2024-T3 and 7075-T6 aluminum alloys. (C) 1998 Elsevier Science Inc. All rights reserved.
\end{abstract}

Keywords: Stochastic modeling; Fatigue crack damage

\section{Introduction}

Stochastic modeling of fatigue crack propagation in metallic materials is a relatively new area of research, and an extensive list of technical literature representing the state of the art is cited by Sobczyk and Spencer (1992), and the special issue of engineering fracture mechanics (Schueller, 1996) presents recent developments in this field. One approach to stochastic modeling of fatigue crack growth is to randomize the coefficients of an established deterministic model to represent material inhomogeneity (Ditlevsen and Olsen, 1986). Another approach is to generate the necessary stochastic information by multiplying the deterministic dynamics of fatigue crack growth with a non-negative random process (Lin and Yang, 1985; Spencer et al., 1989). The process of fatigue crack propagation is thus modeled by nonlinear stochastic differential equations in the Itô setting (Kloeden and Platen, 1995). Specifically, Kolmogorov forward and backward diffusion equations, which require solutions of nonlinear partial differential equations, have been proposed to generate the statistical information required for risk analysis of mechanical structures (Ishikawa et al., 1993; Bolotin, 1989). These nonlinear partial differential equations can only be solved numerically; the computational procedures, however, are computationally intensive as they rely

\footnotetext{
${ }^{*}$ Corresponding author. Tel: +1 814865 6377; fax: +1 814863 4848; e-mail: axr2@psu.edu.
} 
on fine-mesh models using finite-element or combined finite-difference and finite-element methods (Sobczyk and Spencer, 1992). Therefore, although this numerical approach might be useful for making off-line decisions for design analysis and predictive maintenance, it is not sufficiently fast for on-line damage monitoring, failure prognosis, and prediction of remaining service life. Casciati et al. (1992) have analytically approximated the solution of Itô equations by Hermite moments to obtain a probability distribution function of the crack length. To enhance the computational efficiency for on-line execution of the damage estimation and life prediction algorithms, Ray and Tangirala (1996) have developed an algorithm for real-time estimation of fatigue crack damage based on the underlying principle of extended Kalman filtering. In this approach, the first two moments of the stochastic damage state are computed on-line by constructing the stochastic differential equations in the Wiener setting as opposed to the Ito setting.

This paper presents the development of a lognormal distributed crack length (LDCL) model, and verifies the model predictions with the experimental data of fatigue crack growth (Virkler et al., 1979; Ghonem and Dore, 1987) for 2024-T3 and 7075-T6 aluminum alloys.

The LDCL model, presented here, is an enhancement of an earlier model (Ray and Tangirala, 1997), and major differences between these two models are summarized below.

1. The crack growth rate model in the present paper is guaranteed to be non-negative with probability one as compared to the earlier model which yields the non-physical phenomenon of a negative crack growth rate with a small probability.

2. The present model is based on Karhunen-Loève expansion of the crack length process itself in contrast to the earlier model which is based on logarithm of the crack length. This new approach provides an additional free parameter for tuning the probability distribution function.

3. Nonlinear characteristics of the eigenfunctions in the Karhunen-Loève expansion provide better accuracy than the linear representation in the earlier model. This approach allows the model to capture certain nonlinear features of the crack growth statistic. Consequently, model predictions are more accurate.

\section{Modeling of fatigue crack length}

The stochastic model of fatigue crack damage, presented in this paper, is built upon a deterministic model of fatigue crack growth (Newman, 1981) which is based on the principle of short crack growth. The Newman model represents the mean value of the fatigue crack growth process down to micro-cracks of the order of material defect size and has the following form:

$$
\begin{aligned}
& \mathrm{d} \mu_{c}(t)=C\left(\Delta K_{\text {eff }}\right)^{m} \mathrm{~d} t ; \quad \mu_{c}\left(t_{0}\right)>0 ; \quad t \geqslant t_{0}, \\
& \Delta K_{\text {eff }}=\left(S_{\max }-S_{0}\right) \sqrt{\pi \mu_{c}} F,
\end{aligned}
$$

where $\mu_{c}(t) \equiv E\left[\left.c(\omega, t)\right|_{c\left(\omega, t_{0}\right)=\mu_{c}}\right]$ is the expected value of the (time-dependent) crack length process $c(\omega, t)$ conditioned on the initial crack length $c\left(\omega, t_{0}\right)$; a sample of the stressed component is indicated by $\omega$; the time $t$ is expressed in units of number of cycles and $t_{0}$ is the initial time; $\mathrm{d} \mu_{c}$ is the so-called differential of $\mu_{c}$ as commonly used in the fracture mechanics literature (Suresh, 1991); $\Delta K_{\text {eff }}$ is the effective stress intensity factor range; the material-dependent constant $m$ is the slope and the other constant $C$ is the exponential of the intercept of the linear interpolation of the (log scale) $\Delta K_{\text {eff }}-\mathrm{d} \mu_{c} / \mathrm{d} t$ look-up table; $S_{\max }$ is the maximum applied remote stress; $S_{0}$ is the crack opening stress; and $F$ is correction factor for geometrical configuration. Details of this model are reported by Newman (1981). It should be noted, however, that any deterministic fatigue crack growth law can be used in this formulation provided that the average characteristics of the crack growth profile is accurately represented. 
Ray and Tangirala (1997) have modeled the crack length process $c(\omega, t)$ to be implicitly dependent on the (discrete) time parameter, $t$, and the (conditional) variance $\sigma_{c}^{2}(t) \equiv \operatorname{Var}\left[\left.c(\omega, t)\right|_{c\left(\omega, t_{0}\right)=\mu_{c}}\right]$ to be explicitly dependent on the (conditional) expected value $\mu_{c}(t)$ which is directly obtained by solving Eq. (1). Following this approach, the second order statistic of $c(\omega, t)$ is modeled with $\mu_{c}(t)$ as the independent variable in lieu of time, $t$. To this effect, a (dimensionless) continuous function of $\mu_{c}(t)$ is introduced below:

$$
\tau(t):=\left(\frac{\mu_{c}(t)}{\mu_{c}\left(t_{0}\right)}-1\right) \forall t \in\left[t_{0}, t_{f}\right] \text { for } t_{f}<\infty .
$$

The dimensionless parameter $\tau$, which is a monotonically non-decreasing continuous function of $t$, is used as the independent variable in the sequel. In view of the definition in Eq. (3), the stochastic process $\left\{c(\omega, t): t \geqslant t_{0}\right\}$ is denoted as $\left\{c_{\tau}(\omega): \tau \geqslant 0\right\}$.

It is postulated that the random variable $c_{0}(\omega)=\left.c_{\tau}(\omega)\right|_{\tau=0}$ is statistically independent of the random crack increment process defined as

$$
\left.\psi_{\tau}(\omega):=\frac{c_{\tau}(\omega)}{c_{0}(\omega)} \text { for } \tau \geqslant 0 . \quad \text { (Note that } \psi_{0}=1 .\right)
$$

This postulation of independence is based on the rationale that, after an inspection of a structural component, the mean $\mu_{c_{0}} \equiv \mu_{c}\left(t_{0}\right)$ and variance $\sigma_{c_{0}}^{2} \equiv \sigma_{c}^{2}\left(t_{0}\right)$ of the crack length at time $t_{0}$, i.e., at $\tau=0$, are obtained in terms of the measurement data and precision of the measuring instrument. Subsequently, as the structural component undergoes load cycles (i.e., for $\tau>0$ ), the crack propagation process is subjected to material-dependent uncertainties which are independent of measurement uncertainties.

Since the crack length process is a continuous function of $\tau$ in the mean square sense, the crack increment process $\psi_{\tau}$ has a continuous covariance function (Jazwinski, 1970) and therefore can be expressed via Karhunen-Loève expansion (Fukunaga, 1990). The continuous process $\psi_{\tau}$ is discretized at $m$ points beyond $\tau=0$ as an $m$-dimensional random vector $\Psi=\left[\psi_{1} \psi_{2} \ldots \psi_{m}\right]^{\mathrm{T}}$ and $\psi_{j}:=\psi_{\tau_{j}}, j=1,2, \ldots, m$. The covariance matrix $K_{\Psi \Psi}$ of the random vector $\Psi$ is decomposed as

$$
\Psi(\omega):=E[\Psi]+\Phi Z(\omega) ; \quad \operatorname{Cov}(Z)=\Lambda=\operatorname{diag}\left[\lambda_{1} \cdots \lambda_{m}\right],
$$

where the orthogonal matrix $\Phi$ is the modal matrix of $K_{\Psi \Psi}$ and orthogonality of the KarhunenLoève expansion ensures that the random vector $Z=\left[\begin{array}{llll}z_{1} & z_{2} & \ldots & z_{m}\end{array}\right]$ is a set of zero-mean independent random variables. This leads to:

$$
\psi_{k}=E\left[\psi_{k}\right]+\sum_{j=1}^{m}\left(\phi_{k}^{j} z_{j}\right), \quad k=1,2, \ldots, m,
$$

where $\psi_{k}:=\psi_{\tau_{k}}$ and $E\left[\psi_{k}\right]=E\left[c_{\tau_{k}} /\left.c_{0}\right|_{c_{0}=\mu_{c_{0}}}\right]=\mu_{c}\left(\tau_{k}\right) / \mu_{c_{0}}$; and $\phi_{k}^{j} \equiv \phi^{j}\left(\tau_{k}\right)$ is the $k$ th element of the eigenvector $\phi^{j}$. If the first $M$ eigenvalues are dominant, then the random variable $\psi_{k}$ can be approximated as $\hat{\psi}_{k}(M)$ by truncation of the last $(m-M)$ terms in Eq. (6) as

$$
\hat{\psi}_{k}(M) \equiv E\left[\psi_{k}\right]+\sum_{j=1}^{M}\left(\phi_{k}^{j} z_{j}\right), \quad k=1,2, \ldots, m, \quad 1 \leqslant M<m .
$$

Consequently, because of the orthogonality property of the Karhunen-Loève expansion, the covariance of the continuous process, $\hat{\psi}_{\tau}$, can be expressed as

$$
K_{\hat{\psi} \hat{\psi}}\left(\tau_{k}, \tau_{\ell}\right)=\operatorname{Cov}\left(\hat{\psi}_{k}, \hat{\psi}_{\ell}\right)=\sum_{j=1}^{M} \lambda_{j} \phi_{k}^{j} \phi_{\ell}^{j}
$$


having the associated minimum mean square error

$$
\bar{\varepsilon}^{2}(M)=E\left[(\Psi-\hat{\Psi}(M))^{\mathrm{T}}(\Psi-\hat{\Psi}(M))\right]=\sum_{j=M+1}^{m} \lambda_{j}
$$

The statistical information generated from each of the four (discrete) sets of fatigue test data (Virkler et al., 1979; Ghonem and Dore, 1987) show that $\lambda_{1}$ is indeed the dominant eigenvalue as seen in Table 1. The mean square error is in the range of $1.0-3.5 \%$ if $M$ is chosen to be 1, i.e., if only the principal eigenvector, $\phi^{1}(\tau)$, associated with the dominant eigenvalue, $\lambda_{1}$, is used to model the process $\psi_{\tau}$. A constitutive relationship is proposed for $\phi^{1}(\tau)$ as a continuous function of $\tau$ :

$$
\phi^{1}(\tau)=\left(\sqrt{Q_{c} / \lambda_{1}}\right)\left(\frac{\mathrm{e}^{\tau \xi}-1}{\xi}\right)
$$

Setting $M=1$, the covariance in Eq. (8) is modified by Eq. (10) as

$$
K_{\hat{\psi} \hat{\psi}}(\tau+\theta, \tau) \approx Q_{c}\left(\frac{\mathrm{e}^{\xi(\tau+\theta)}-1}{\xi}\right)\left(\frac{\mathrm{e}^{\tau \xi}-1}{\xi}\right)
$$

and $K_{\psi \psi}(\tau, \tau) \approx K_{\hat{\psi} \hat{\psi}}(\tau, \tau)=\sigma_{\psi}^{2}(\tau)$ is readily obtained from Eq. (11). Based on statistical fatigue test data, the model parameters $\xi$ and $Q_{c}$ are found to have the following physical characteristics:

- $\xi$ is material-dependent but it is independent of the peak stress and stress ratio to which the specimens were subjected.

- $Q_{c}$ is both stress-dependent and material-dependent.

Remark 1. Separation of the stochastic process $\left(\psi_{\tau}-E\left[\psi_{\tau}\right]\right)$ into (deterministic) $\tau$-dependent and (random) $\omega$-dependent variables follows by choosing $M=1$ in Eq. (7). If $M>1$ is chosen, then the additional terms to be included in the model of Eq. (11) will act as small perturbations.

Chi-square tests conducted on the fatigue test data at different instants (i.e., cycles) reveal that the crack length distribution can be approximated as (non-stationary) lognormal within a significance level of $5 \%$. Based on this information, a lognormal random variable is defined as

$$
\eta(\omega) \equiv\left(\sqrt{\frac{Q_{c}}{\lambda_{1}}}\right) z_{1}(\omega) ; \quad E[\eta]=0 \text { and } \operatorname{Var}[\eta]=Q_{c} .
$$

Setting $M=1$ in Eq. (8), and following Eqs. (3), (6), (10) and (12), the fatigue-induced crack length is modeled as

$$
\psi_{\tau}(\omega)=\frac{c_{\tau}(\omega)}{c_{0}(\omega)}=\left(\tau+1+\left(\frac{\mathrm{e}^{\tau \xi}-1}{\xi}\right) \eta(\omega)\right) \Rightarrow \mathrm{d} \psi_{\tau}(\omega)=\frac{\mathrm{d} c_{\tau}(\omega)}{c_{0}(\omega)}=\left(1+\mathrm{e}^{\tau \xi} \eta(\omega)\right) \mathrm{d} \tau
$$

Table 1

Dominant eigenvalue of the covariance matrix

\begin{tabular}{ll}
\hline Statistical data set of fatigue crack length & $\lambda_{1} / \sum_{j=1}^{m} \lambda_{j}$ for any $m \geqslant 20$ \\
\hline Virkler et al. & $\sim 0.965$ \\
Ghonem and Dore \#1 & $\sim 0.990$ \\
Ghonem and Dore \#2 & $\sim 0.968$ \\
Ghonem and Dore \#3 & $\sim 0.988$ \\
\hline
\end{tabular}


Remark 2. The dynamics of fatigue crack growth is represented in Eq. (13) in terms of the dimensionless parameter $\tau$ which is a monotonically increasing function of time (or cycles) as defined in Eq. (3).

The physical phenomena, $\mu_{c_{0}}>0, c_{0}(\omega)>0, \mathrm{~d} \mu_{c}(\tau) \geqslant 0$, and $\mathrm{d} c_{\tau}(\omega) \geqslant 0 \forall \omega \forall \tau \geqslant 0$, imply that the model in Eq. (13) must satisfy the inequality constraint: $\left(\mathrm{d} \psi_{\tau}(\omega) / \mathrm{d} \tau \geqslant 0 \forall \omega\right.$. Therefore,

$$
\inf _{\omega}\left(\frac{\mathrm{d} \psi_{\tau}(\omega)}{\mathrm{d} \tau}\right) \geqslant 0 \Rightarrow \inf _{\omega} \eta(\omega) \geqslant-\mathrm{e}^{-\xi \tau}
$$

It suffices to satisfy the inequality in Eq. (14) for a given critical crack length, $\mu_{c_{f}}$, at which the service life of the stressed structure is considered to be completely expended. In general, $\mu_{c_{f}}$ depends on several factors including the geometry and dimensions of the stressed structure, the allowable factor of safety, and sensitivity and resolution of the inspection equipment. Following Eq. (3), the dimensionless parameter $\tau_{f}$ is defined as

$$
\tau_{f}:=\frac{\mu_{c_{f}}}{\mu_{c_{0}}}-1
$$

ollowing Eq. (13), if $\mathrm{d} \psi_{\tau}(\omega) / \mathrm{d} \tau=0$ for some $\omega$, then $\left(\mathrm{d} \psi_{\tau}(\omega) / \mathrm{d} \tau\right) \geqslant 0 \forall \tau \in\left[0, \tau_{f}\right)$ for the same $\omega$. Therefore, setting $\inf _{\omega}\left(\mathrm{d} \psi_{\tau}(\omega) / \mathrm{d} \tau\right)_{\tau=\tau_{f}}=0$ guarantees non-negative crack growth rate for all cracks less than the critical crack length $\mu_{c_{f}}$.

Remark 3. In general, $\mu_{c}$ represents the critical crack length beyond which the crack growth rate becomes very large rapidly leading to complete rupture (Ishikawa et al., 1986). Therefore, $\mu_{c_{f}}$ (and hence $\tau_{f}$ ) depends on several factors including the geometry and dimensions of the stressed structure, the allowable factor of safety, and sensitivity and resolution of the inspection equipment.

The zero-mean lognormal variable $\eta(\omega)$ in Eq. (12) is expressed in terms of a Gaussian variable, $y(\omega)$, as

$$
\eta(\omega)=\mathrm{e}^{y(\omega)}-E\left[\mathrm{e}^{y(\omega)}\right] ; \quad y \sim N\left(m, \sigma^{2}\right) .
$$

The following two coupled algebraic equations are derived from Eqs. (12), (14) and (16)

$$
\begin{aligned}
& \inf _{\omega} \eta(\omega)=-\exp \left(-\xi \tau_{f}\right) \Rightarrow m+\frac{\sigma^{2}}{2}=-\xi \tau_{f}, \\
& \exp \left(2\left(m+\frac{\sigma^{2}}{2}\right)\right)\left(\exp \left(\sigma^{2}\right)-1\right)=Q_{c} .
\end{aligned}
$$

Eqs. (17) and (18) are solved simultaneously to yield

$$
\sigma^{2}=\ln \left(1+Q_{c} \exp \left(2 \xi \tau_{f}\right)\right), \quad m=-\left(\xi \tau_{f}+\frac{\sigma^{2}}{2}\right) .
$$

Given $\xi, Q_{c}$ and $\tau_{f}$, the unknowns $m$ and $\sigma^{2}$ in Eq. (19) can be evaluated to specify the probability density function (pdf) of the Gaussian random variable $y(\omega)$ in Eq. (16). This information, in turn, prescribes the lognormal pdf of $\eta(\omega)=\mathrm{e}^{y(\omega)}-\mathrm{e}^{m+\left(\sigma^{2} / 2\right)}$ as

$$
f_{\eta \mid c_{0}}\left(\left.\theta\right|_{\mu_{c_{0}}}\right)= \begin{cases}\mathrm{e}^{\frac{1}{-2 \sigma^{2}}\left(\ln \left(\theta+\mathrm{e}^{m+\left(\sigma^{2} / 2\right)}\right)-m\right)^{2}} /\left(\theta+\mathrm{e}^{m+\left(\sigma^{2} / 2\right)}\right) \sqrt{2 \pi \sigma^{2}}, & \theta \geqslant-\mathrm{e}^{m+\left(\sigma^{2} / 2\right)}, \\ 0 & \text { otherwise. }\end{cases}
$$

Following Eqs. (4), (13) and (20), the pdf of $\psi_{\tau}(\omega)=c_{\tau}(\omega) / c_{0}(\omega)=\left(1+\tau+\left(\left(\mathrm{e}^{\xi \tau}-1\right) / \xi\right) \eta(\omega)\right)$ is 


$$
f_{\psi \mid c_{0}}\left(\theta ;\left.\tau\right|_{\mu_{c_{0}}}\right)=\left\{\begin{array}{l}
\frac{\exp \left(\left(\ln \left(\left(\frac{(\theta-\tau-1) \xi}{\mathrm{e}^{\xi \tau}-1}\right)+\mathrm{e}^{m+\left(\sigma^{2} / 2\right)}\right)-m\right)^{2} /\left(-2 \sigma^{2}\right)\right)}{\left(\left(\frac{(\theta-\tau-1) \xi}{\mathrm{e}^{\xi}-1}\right)+\mathrm{e}^{m+\left(\sigma^{2} / 2\right)}\right)\left(\frac{\mathrm{e}^{\xi \tau}-1}{\xi}\right) \sqrt{2 \pi \sigma^{2}}} \\
\text { for } \theta \geqslant \tau+1-\frac{\mathrm{e}^{m+\left(\sigma^{2} / 2\right)}\left(\mathrm{e}^{\xi \tau}-1\right)}{\xi}, \\
0 \quad \text { otherwise. }
\end{array}\right.
$$

\section{Model verification}

The stochastic damage model is verified using the statistical data of fatigue crack propagation for 2024-T3 aluminum alloy (Virkler et al., 1979) and for 7075-T6 aluminum alloy (Ghonem and Dore, 1987), in which the tests were conducted under different constant load amplitudes at ambient temperature. The Virkler data set was generated at a single load level with peak nominal stress of $60.33 \mathrm{MPa}(8.75 \mathrm{ksi})$ and stress ratio $R=0.2$. Ghonem and Dore data sets were generated at three different load levels: (i) Set 1 with peak nominal stress of $70.65 \mathrm{MPa}(10.25 \mathrm{ksi})$ and $R=0.6$; (ii) Set 2 with peak nominal stress of $69.00 \mathrm{MPa}(10.00 \mathrm{ksi})$ and $R=0.5$; and (iii) Set 3 with peak nominal stress of $47.09 \mathrm{MPa}(6.83 \mathrm{ksi})$ and $R=0.4$. The four plates in Fig. 1 display comparisons of model-predicted variance of the crack length process with the experimental data as a function of load cycles for 2024-T3 and 7075-T6 aluminum alloys. The model predictions are in very close agreement with the respective experimental results derived from each of the four data sets.

The material-dependent parameter $\xi$, introduced in Eq. (10), is independent of the peak stress and stress amplitude to which the specimens were subjected. Note that $\xi=0.25$ for $2024-\mathrm{T} 3$ for the single data set and $\xi=0.75$ for 7075 -T6 for all three data sets. Apparently, the stochastic model parameter $Q_{c}$ is dependent on the material and both peak stress and $R$ as seen in each of the four plates in Fig. 1. It should be noted that each of the above data sets was generated with an initial crack length of $\sim 9 \mathrm{~mm}$ (i.e., $\mu_{c_{0}} \approx 9 \mathrm{~mm}$ and $\sigma_{c_{0}}^{2} \approx 0$ ) at ambient temperature in the laboratory environment. It is likely that $Q_{c}$ is strongly dependent on the environmental condition and also is a function of $\mu_{c_{0}}$. The results, obtained from the lognormal-distributed crack length (LDCL) model, are comparable or superior in accuracy to those generated by the lognormal-distributed crack growth rate (LDCGR) model of Spencer et al. (1989) that requires numerical solution of the Kolmogorov diffusion equations.

It is well known (Kloeden and Platen, 1995) that real-time solution of the partial differential equations encountered in the Kolmogorov equations is not computationally feasible even on mainframe computers. In contrast to the LDCGR model, the LDCL model requires the solution of only a few algebraic identities which can be conveniently executed in real time on a microcomputer. However, it should be noted that the Kolmogorov equations yield the conditional density as a function of time while the LDCL model, in the present form, generates the two-parameter lognormal distribution function from the first two moments of crack length.

\section{Summary and conclusions}

This paper presents a stochastic model of fatigue crack length process in metallic materials for damage estimation and life prediction of stressed structures. The model equations are based on the underlying principle of the Karhunen-Loève expansion of the crack length covariance. The 

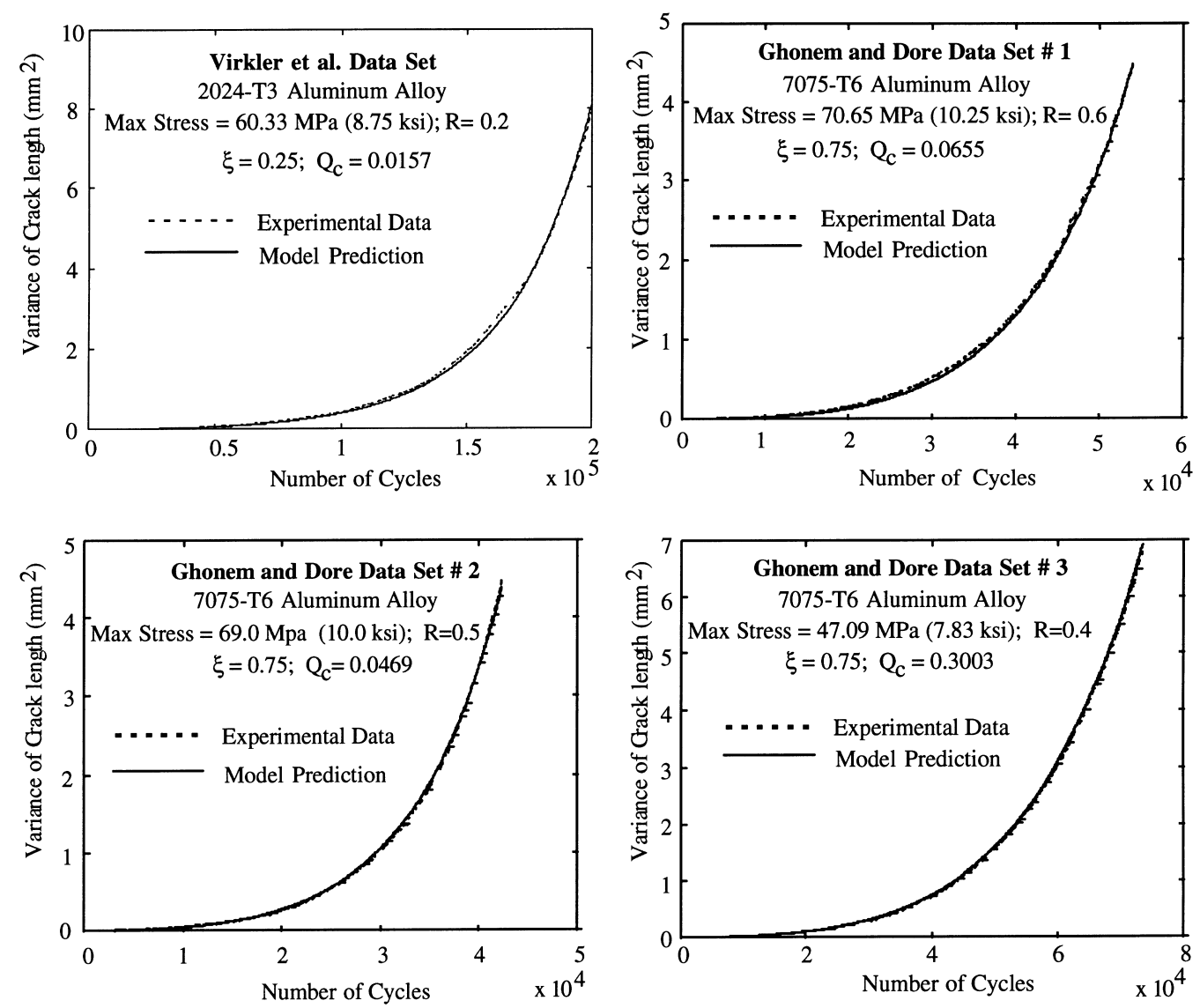

Fig. 1. Crack length variance for 2024-T3 and 7075-T6 aluminum alloys.

(non-stationary) statistics of the crack growth process are obtained without solving the extended Kalman filter equation in the Wiener integral setting or the Kolmogorov forward equation in the Itô integral setting. The model, presented in this paper, also guarantees non-negativity of the crack growth rate. The mean of the time-dependent crack length can be obtained from one of the several models that are reported in the literature. The stochastic model calculates the variance of crack length as a solution of algebraic identities that is computationally much faster than numerically solving the stochastic differential equations.

The stochastic model of fatigue crack length, presented in this paper, is formulated under the postulation that the crack length has the lognormal distribution instead of the more common assumption that the crack growth rate is lognormally distributed. Based on this postulation, probability distribution function of the (non-stationary) crack length process can be generated from the first two moments. This information suffices to determine, at a given level of confidence, the (time-dependent) remaining life of mechanical structures subjected to a specified anticipated load profile (Ray and Tangirala, 1996). The increased speed of the proposed crack length model makes it ideally suited to real-time damage monitoring and failure prognostic applications.

The proposed lognormal-distributed crack length (LDCL) model has been verified with experimental fatigue crack growth data of 2024-T3 and 7075-T6 aluminum alloys at different levels of constant amplitude load excitation. Extension of this model to load excitation of varying amplitude is a subject of current research. While the stochastic modeling approach, presented in this 
paper, focuses on the inherent material uncertainties, there are two other major sources of uncertainties, namely, random loading and unknown initial conditions which have not been considered here. A unified model that accounts for all three primary sources of uncertainties in crack growth needs to be developed before practical applications become viable.

Potential applications of the fatigue crack model include: (i) equipment readiness assessment and failure diagnosis and prognosis of operating machinery based on current condition and projected; (ii) generation of alerts and warnings for operational support and safety enhancement; and (iii) formulation of real-time decision policies for information-based maintenance.

\section{Acknowledgements}

The authors are grateful to Professor B.M. Hillberry of Purdue University and Professor H. Ghonem of University of Rhode Island for providing the experimental data. The work presented in this paper has been supported in part by the National Science Foundation under grant nos. DMI-9424587 and CMS-9531835.

\section{References}

Bolotin, V.V., 1989. Prediction of Service Life for Machines and Structures. ASME, New York.

Casciati, F., Colombi, P., Farvelli, L., 1992. Fatigue crack size probability distribution via a filter technique. Fatigue and Fracture of Engineering Materials and Structures 15 (5), 463-475.

Ditlevsen, O., Olsen, R., 1986. Statistical analysis of the virkler data on fatigue crack growth. Engineering Fracture Mechanics 25 (2), 177-195.

Fukunaga, K., 1990. Introduction to Statistical Pattern Recognition, 2nd ed. Academic Press, Boston, MA.

Ghonem, H., Dore, S., 1987. Experimental study of the constant probability crack growth curves under constant amplitude loading. Engineering Fracture Mechanics 27, 1-25.

Ishikawa, H., Tsurui, A., Tanaka, A.H., Ishikawa, H., 1993. Reliability assessment based upon probabilistic fracture mechanics. Probabilistic Engineering Mechanics 8, 43-56.

Jazwinski, A.H., 1970. Stochastic Processes and Filtering Theory. Academic Press, New York.

Kloeden, P.E., Platen, E., 1995. Numerical Solution of Stochastic Differential Equations. Springer, Berlin.

Lin, Y.K., Yang, J.N., 1985. A stochastic theory of fatigue crack propagation. AIAA Journal 23 (1), 117-124.

Newman, J.C., 1981. A crack-closure model for predicting fatigue crack growth under aircraft loading. Methods and Models for Predicting Fatigue Crack Growth under Random Loading, ASTM STP 748, 53-84.

Ray, A., Tangirala, S., 1996. Stochastic modeling of fatigue crack dynamics for on-line failure prognostics. IEEE Trans. Control Systems Technology 4 (2), 443-451.

Ray, A., Tangirala, S., 1997. A nonlinear stochastic model of fatigue crack dynamics. Probabilistic Engineering Mechanics 12 (1), 33-40.

Schueller, G.I. (Ed.), 1996. Reliability of structural and mechanical components under fatigue (special issue). Engineering Fracture Mechanics 53 (5).

Sobczyk, K., Spencer Jr., B.F., 1992. Random Fatigue: Data to Theory. Academic Press, Boston, MA.

Spencer, B.F., Tang, J., Artley, M.E., 1989. A stochastic approach to modeling fatigue crack growth. AIAA Journal 27 (11), 1628-1635.

Suresh, S., 1991. Fatigue of Materials. Cambridge University Press, Cambridge, UK.

Virkler, D.A., Hillberry, B.M., Goel, P.K., 1979. The statistical nature of fatigue crack propagation. ASME Journal of Engineering Materials and Technology 101 (2), 148-153. 